Florida International University FIU Digital Commons

3-28-1994

\title{
Infants' detection of synchrony between sounds and pauses in the movement of an object
}

Linda Alberga

Florida International University

DOI: $10.25148 /$ etd.FI13101562

Follow this and additional works at: https://digitalcommons.fiu.edu/etd

Part of the Child Psychology Commons

\section{Recommended Citation}

Alberga, Linda, "Infants' detection of synchrony between sounds and pauses in the movement of an object" (1994). FIU Electronic Theses and Dissertations. 1188.

https://digitalcommons.fiu.edu/etd/1188

This work is brought to you for free and open access by the University Graduate School at FIU Digital Commons. It has been accepted for inclusion in FIU Electronic Theses and Dissertations by an authorized administrator of FIU Digital Commons. For more information, please contact dcc@fiu.edu. 
FLORIDA INTERNATIONAL UNIVERSITY

Miami, Florida

INFANTS' DETECTION OF SYNCHRONY BETWEEN SOUNDS AND PAUSES IN THE MOVEMENT OF AN OBJECT

A thesis submitted in partial satisfaction of the requirements for the degree of

MASTER OF SCIENCE

IN

PSYCHOLOGY

by

Linda Alberga 
To: Dean Arthur W. Herriott

College of Arts and Sciences

This thesis, written by Linda Alberga, and entitled Infants' Detection of Synchrony Between Sounds and Pauses in the Movement of an object, having been approved in respect to style and intellectual content, is referred to you for judgement.

We have read this thesis and recommend that it be approved.

William Kurtines

Janat dafker

Lorraine Bahrick, Major Professor

Date of Defense: March 28, 1994

The thesis of Linda Alberga is approved.

Dean 3 fthur $W$. Herriott

collqge of Arts and Sciences

Dr. Richard L. Campbell

Dean of Graduate studies

Florida International University, 1994 


\title{
ABSTRACT OF THE THESIS
}

INFANTS' DETECTION OF SYNCHRONY BETWEEN SOUNDS AND PAUSES IN THE MOVEMENT OF AN OBJECT

by

\section{Linda Alberga}

\author{
Florida International University, 1994 \\ Professor Lorraine Bahrick, Major Professor
}

The present study investigated the development of sensitivity to temporal synchrony between sounds of impact and pauses in the movement of an object by infants of $21 / 2$, 4 and 6 months of age. Ninety infants were tested across four experiments with side-by-side videos of a red and white square and $\mathrm{a}$ blue and yellow triangle along with a centralized soundtrack which was synchronized with only one of the films. This preference phase was then followed by a search phase, where the two films were accompanied by intermittent bursts of the soundtrack from each object. Twomonth-olds showed no evidence of matching films and soundtracks on the basis of synchrony, however 4-month-olds looked more on the second block of trials to the object which paused when the sound occurred and directed more first looks during the preference phase to the matching object. Six-month-olds demonstrated significantly more first looks to the mismatched object during the search phase only. These 
results suggest that infants relate impact sounds with synchronous pauses in continuous motion by the age of four months. 
II. LITERATURE REVIEW

Perception of audio-visual tempo and synchrony

Perception of face-voice relations . . . . . .

Perception of temporal microstructure . . . . . . 13

Intermodal Learning . . . . . . . . . . . . . . 18

Perception of Object Motion . . . . . . . . . . 24

III. EXPERIMENTAL DESIGN . . . . . . . . . . . . . . . 31

Experiment 1 . . . . . . . . . . . . . . . . . . 31

Method . . . . . . . . . . . . . . . . 32

Results and Discussion . . . . . . . . . . . 37

Experiment 2 . . . . . . . . . . . . . . . . . . 43

Method . . . . . . . . . . . . . . . . . 44

Results and Discussion . . . . . . . . . . . . 45

Experiment 3 . . . . . . . . . . . . . . . 47

Method . . . . . . . . . . . . . . . 47

Results. . . . . . . . . . . . . . . . . . 48

Comparison Across Experiments . . . . . . . . 54

Discussion . . . . . . . . . . . . . . . . 55

Experiment 4 . . . . . . . . . . . . . . . 56

Method . . . . . . . . . . . . . . . . 56

Results . . . . . . . . . . . . . . . . . . 57

Comparison Across Experiments . . . . . . . . . 58

Discussion . . . . . . . . . . . . . . 58

IV. GENERAL DISCUSSION . . . . . . . . . . . . . . . . . . .

LIST OF REFERENCES . . . . . . . . . . . . . . . . . . . . . 65

TABLES . . . . . . . . . . . . . . . . . . . . . . . . . . .

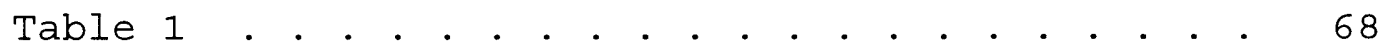

Table 2 . . . . . . . . . . . . . . . . . . . . 69

Table 3 . . . . . . . . . . . . . . . . . . 70

Table 4 . . . . . . . . . . . . . . . . . . . 71

Table 5 . . . . . . . . . . . . . . . . . . 72

Appendix . . . . . . . . . . . . . . . . . . . . . . . . . 73 


\section{Chapter 1 \\ INTRODUCTION}

The development of intermodal perception has become an area of increasing interest among researchers in infancy. Intermodal perception refers to the ability to perceive as a whole information obtained from two or more senses simultaneously. Interest in this issue was sparked by two conflicting theoretical positions. The traditional association theorists (Birch and Lefford, 1963; Bryant, 1974) argued that infants received stimulation to the senses through different channels and that development consisted of gradually learning to integrate these separate sensory experiences. However, before this integration occurs, the infant perceives nothing but unrelated streams of light and sound. Piaget (1952) also held a similar integration perspective. He believed that the infant learned to integrate the sensations produced through different sense modalities. However, in contrast to the association theorists, Piaget proposed that perception and knowledge evolved not through the process of association but as a result of the child acting on his or her environment. According to Piaget the infant plays an active role in his/her own perceptual development and that development is dependent on the construction of structures which are not innate in the child but which change and develop through a 
process of assimilation and accommodation. Assimilation is the process by which an organism integrates new information into the already existing body of information or schemes whereas accommodation is the process of changing the already existing scheme to integrate new information.

In contrast to these viewpoints, Gibson (1969) proposed that infants from birth are capable of directly perceiving audio-visual information in the environment without needing to associate stimulation across the senses or needing to invent or construct knowledge as proposed by Piaget. Rather she believes that the basis for perception is the organism's ability to detect invariants, that is, properties which remain constant across changing stimulation from objects and events. Further she proposed that the senses are unified at birth and that the task of the infant is to differentiate information from the different senses. Infants from birth are equipped with the ability to detect invariants and as they get older they refine this capability by differentiating increasingly finer levels of stimulation. The purpose of this paper is to examine how infants respond to relationships between the optic and acoustic stimulation which specify properties of objects in the world. More specifically the paper provides a developmental outlook on whether 2-month-old, 4-month-old and 6-month-old infants are able to detect a correspondence between sounds 
of impact and pauses in the movement of an object. 


\section{Chapter 2}

\section{Literature Review}

The intermodal preference method which was developed by Spelke (1976) has been useful in investigating the infant's detection of correspondences between visual events and their corresponding sounds. In this method, infants typically view two filmed events side by side, while a soundtrack corresponding to one of the events is played from a centrally located speaker. If infants perceive the sound as related to the appropriate event, they should look longer and more frequently at that event. A search phase typically follows the preference procedure. During this phase, infants hear intermittent presentations of the soundtracks from each film while both objects are in view. The measure of interest is the direction of the infant's first look at the onset of each soundtrack presentation. If infants learned the correct object-sound relationship during the preference phase then they should demonstrate this knowledge in the search phase by looking first more often to the object which was synchronized with that sound in the preference phase. Using this method, it has been demonstrated that infants by the age of $41 / 2$ months detect temporal synchrony relations (Spelke, 1979; 1981; Spelke \& Cortelyou, 1980; Bahrick, 1983) as well as the common tempo of action uniting the visual and acoustic stimulation of an event (Spelke, 1979). 


\section{Perception of audio-visual tempo and synchrony}

Spelke (1979) demonstrated that 4-month-old infants can perceive the unity of an audible and visible display by detecting the temporal synchrony between the object's sounds and its impacts to a surface as well as the common tempo of action uniting the visible and acoustic stimulation. This was demonstrated in a series of three experiments using the intermodal preference method. In experiment 1, 4-month-old infants were presented with two films simultaneously, each with a different puppet moving at a different speed. One puppet was moved at a rate of one bounce per two seconds while the other was moved at a rate of one bounce every second. A different percussive sound accompanied each puppet's impact to the ground. In one 60-sec trial, the impacts of one puppet were synchronized with a "thump" sound and in the other $60-$ sec trial the impacts of the second puppet were synchronized with a "gong" sound. In this experiment each sound occurred at the same tempo as the impacts of one object and was simultaneous with those impacts. Results indicated that infants looked first and more quickly to the event in synchrony. These results support the author's hypothesis that infants are sensitive to the temporal patterning of sights and sounds when the object's sounds and its impacts are synchronized and share a common tempo. However, it is possible that infants may have 
detected a relationship between the sounds and impacts on the basis of a common tempo or on the basis of synchrony between visual and acoustic impacts. Spelke sought to clarify this issue in Experiments 2 and 3 where she examined infants' detection of synchrony and tempo in isolation. Experiment 2 investigated tempo while holding synchrony constant. This was accomplished by not presenting the sounds and impacts simultaneously. During the preference trials, each soundtrack was played out of phase with the impacts of each object and the objects were moved out of phase with each other. Only the rate of movement united the matching soundtrack with the filmed object. The results of the preference episode indicated that there was no preference to look longer at the acoustically specified event. However during the search episode that followed there were more first and eventual looks toward the acoustically specified event than toward the non-specified event. Therefore 4month-olds seem to detect the common tempo uniting audible and visual stimulation.

Experiment 3 investigated infants' detection of synchrony while keeping tempo constant. During the preference trials, the infants were presented with two animals moving at the same rate and with one film synchronized with the thump sound and the other with the gong sound. Only the synchrony of the sound and its impacts 
united each soundtrack with its appropriate film. The results of the preference episode, indicated that infants exhibited a visual preference for the acoustically synchronized event but this preference was only reliable when data from both sessions were combined. In the search episode, infants looked first to the synchronized event on more trials. These results show that 4 -month-old infants can detect auditory visual correspondences when the sounds and impacts are synchronized and when the synchronized and non synchronized objects moved at the same rate. Spelke (1979) provided evidence that infants can detect at least two types of invariants. They can detect common tempo and the synchrony of soundbursts with the visible impacts of objects.

Humphrey and Tees (1980) also examined infants' perception of audio-visual tempo and synchrony using two visual displays of fast versus slow flashing lights side by side and with tones which were temporally synchronized to one of the displays. The visual display and corresponding tones differed in temporal rate by a factor of four. Infants of 3,7 and 10 months of age were presented with four sixtysecond trials, two slow and two fast tempos. No evidence was found for preferential looking to the sound-specified visual pattern for the 3 and 7-month-olds, however, the 10-monthold group looked more often at the visual pattern with the 
faster temporal rate regardless of position or tempo of the accompanying sound. These results were only marginally significant. In sum, this study provided no evidence for the detection of synchrony but it provided some evidence for the visual detection of tempo in older infants. The author's lack of success in demonstrating sensitivity to synchrony in the 3 and 7-month-old groups may have been due to the fact that the visual stimuli selected for this study may have been too similar to each other. Therefore it was more difficult for the younger infants to discriminate between the two displays on the basis of tempo.

Lewkowicz (1985) also addressed the issue of tempo by examining whether infants could match auditory and visual stimuli on the basis of temporal frequency. This was demonstrated by presenting 4 -month-olds with random check patterns flashing at different rates both in the presence and absence of concurrent sound. No evidence was found of bisensory matching of the rate of the sound with that of the light. A second and third study were conducted to see whether matching would occur if both frequency and intensity were varied or if intensity was varied and temporal frequency was kept constant. No evidence of bisensory matching was found in either condition. However from both experiments 2 and 3 it is apparent that the presence of a high frequency sound resulted in looking towards a low 
frequency light. In contrast to the findings of Spelke (1979) and Humphrey, Tees and Werker (1979), Lewkowicz was unable to show that infants could match on the basis of temporal frequency. The failure to exhibit matching may have been due to the unnatural nature or high frequency of the stimuli used. The displays used in this study were tones and flashing checks rather than moving and sounding objects like those used by Bahrick's and Spelke's studies. Infants may also have been unable to detect the sound specified visual patterns because the displays were too similar to each other. Also the author seems to have overlooked the issue of synchrony as there is no mention as to whether the check patterns were presented synchronously with the tone.

Although this study provides no evidence for the detection of temporal relations, it is clear from the work of Spelke (1979) that infants as young as 4 months old are able to unite an audio and a visual event on the basis of common temporal relations. Furthermore, Humphrey and Tees (1980) provide some evidence for the detection of tempo in older infants.

Menten and Cohen (1979) have replicated the findings of Spelke (1979) by showing that 4-month-old infants can detect synchrony between sights and sounds. The first experiment was conducted by presenting infants with two films of a toy kangaroo side by side, moving at the same rate of motion but 
out of phase with each other by either one half of a second or one second. Here the soundtrack was synchronous with only one film. This procedure eliminated the confound between tempo and synchrony as tempo was held constant like in Spelke's study. Each infant received eight 30-sec trials with the soundtrack appropriate to one film. Four trials were presented out of phase by one half of a second and four were out of phase by one second. Results indicated that the infants looked more to the synchronous film than to the asynchronous film. However, only the male infants fixated the synchronous films significantly more than chance. These results indicate that male infants detected synchrony.

As this was one of the earliest studies to provide evidence of sex differences in the detection of temporal correspondences, the authors did a second experiment using a different procedure in an effort to replicate their results. In contrast to Experiment 1, the second experiment was conducted utilizing a successive presentation procedure of the sound-film pairings rather than a simultaneous one. That is, infants viewed a single event with the soundtrack alternately in synchrony and out of synchrony with the film. Half the infants viewed the soundtrack in synchrony with the film while the other half viewed the film one second out of synchrony with the sound. The results indicated that the 4month-old males preferred the synchronous presentation over 
the asynchronous. The authors concluded that 4-month-old male infants can perceive synchrony and can discriminate it from asynchrony. Overall these experiments provide partial support for the hypothesis that 4-month-old infants can perceive auditory visual temporal relations.

Mendelson and Ferland (1982) also demonstrated that infants by 4 months of age were sensitive to the common rhythm uniting the sight of a puppet opening and closing its mouth with the sounds it made. The authors concluded that infants as young as four months perceive the equivalence between auditory and visual temporal information. However, these results appear to provide suggestive but not conclusive evidence as cross-modal transfer was only obtained when infants heard the regular rhythm and not when they heard an irregular rhythm.

Perception of face-voice relations

Other research has demonstrated that infants are sensitive to the congruence between lip movements and speech sounds (Dodd, 1979). Dodd (1979) found that when infants were presented with the face of an experimenter reciting nursery rhymes they attended significantly less to the outof-synchrony presentation than to the in-synchrony presentation. This finding was interpreted as an indication that young infants are aware when sound and lip movements do not match even though they do not understand what is being 
said. However, it is possible that the results could be based on the congruence between the shape of the mouth and type of sound uttered.

The findings of Dodd's (1979) study have been corroborated by Spelke and Cortelyou (1980) who presented infants with films of the faces of two women side by side. Infants heard one voice at a time while both films were displayed and each voice was played concurrently with both faces. The synchronized voice of each woman was played through a centrally located speaker. This procedure ensured that infants would be unable to use the spatial location of the voice to guide their discovery of the face-voice relationship. Also the face-voice pairings were unknown to the infants. Therefore only the temporal synchrony of speech and facial movements could tie each voice to each person. The results indicated that during the preference session infants looked more to the "speaking" synchronous woman, while in the search phase infants looked first and looked more often to the woman whose voice they heard. The authors concluded that by detecting the synchrony of speech and facial movements infants can determine that a particular voice belongs to a particular face. Another possible explanation of these results may be that rather than responding to the synchrony of speech and facial movements infants might also have been able to match the simultaneous 
occurrence of speaking and moving to the pattern of pausing and resting in the displays which they saw and heard. Like Dodd's research infants may have responded to the synchrony of speech sounds and articulatory movements.

In summary, these findings suggest that infants appear to be sensitive to the synchrony of certain characteristics of speech with certain visible movements of the speaker. However other information may have also contributed to the infant's ability to match such as the correspondence between the shape of the mouth and the motion of the sounds produced or the rhythmical pattern of speech.

Other researchers such as Pickens, Field, Nawrocki, Martinez, Soutoullo and Gonzalez (1992) have compared preterm infants versus fullterm infants between the ages of 3 and 7 months on their ability to match faces and voices. Their results indicated that fullterm infants displayed evidence of face-voice matching at 3 and 7 months but not at 5 months of age. However preterm infants did not show evidence of detecting face-voice synchrony. The findings for the fullterm infants are suggestive of a U-shaped developmental curve of auditory-visual matching as a function of age.

Perception of temporal microstructure

Other investigators have focused on the infant's perception of objects of different substances and objects of 
different compositions (Bahrick, 1983; 1987). Bahrick (1983)

looked at infants' audiovisual perception of rigidity or elasticity of substance. This was assessed by presenting 4 1/2-month-old infants with two films side by side of sponges squishing and wooden blocks banging in an erratic pattern and with the soundtrack to one of the films coming from a centrally placed speaker. The results indicated that infants looked significantly longer at the sound synchronized and appropriate film. From the results of this experiment it can be concluded that infants may have detected the synchrony between the sights and sounds of impact or they may have detected the temporal information (microstructure) specifying the substance of the objects or both these factors. Therefore experiments 2,3 and 4 were conducted to determine this.

In experiment 2 synchrony and substance information were placed in conflict by putting each soundtrack in synchrony with the wrong film. Infants now showed no preference for either film suggesting that neither synchrony nor substance was sufficient by itself to allow the relationship to be detected.

In experiment 3, infants' detection of information specifying the substance of the object in the absence of synchrony was examined by presenting the sound and the films successively. That is, the soundtrack was played first and 
then the films were shown simultaneously and without any accompanying sound. Infants looked more to the film whose soundtrack they had heard previously. These results suggested that infants detected the temporal structure specifying the object substance even in the absence of a synchronized presentation of the soundtrack and the film.

In experiment 4, infants' detection of temporal synchrony information in the absence of substance information was investigated by presenting two versions of the same event side by side and with the soundtrack synchronized to one of them. Results indicated a reliable looking preference for the film that was out of synchrony with the soundtrack during the first viewing of the preference phase. However the infants did not search reliably in this experiment. Although the infants showed a preference for the films that were out of synchrony with the soundtrack, this experiment still provides evidence of the infant's detection of temporal synchrony information as the films differed only in their temporal relation to the soundtrack. Therefore any differential looking must have been on that basis. These experiments demonstrated that infants detected the temporal information specifying the object's rigidity and elasticity as well as the temporal synchrony between the sights and sounds in these events. However because these events were natural and possibly 
familiar it is possible that infants might have known beforehand of the relevant auditory-visual correspondences.

Bahrick (1987) by using preferential looking to a sound specified film attempted to determine at what age infants detected audiovisual relations on the basis of synchrony or temporal microstructure which can specify properties of an object such as its substance, number and composition. This was investigated by presenting infants of $3,41 / 2$ and 6 months with films of a single object (large marble) and a compound object (a group of small marbles) colliding against a surface. In one experimental condition, infants viewed a film of a single large marble and a film of a group of small marbles side by side and heard a soundtrack which was synchronized to the motions and appropriate to the composition of one of the events. During the search phase, the films were played out of phase with one another. The results indicated that the 6-month-old infants showed a significant proportion of first looks to the sound specified film during the search phase. However no significant effects were found for the 3 and 4 1/2-month old infants. However, the infant's search performance showed a linear improvement across age. Infants may have responded on the basis of temporal synchrony which united the films and their soundtracks or they may have responded to the temporal microstructure which specified the composition of the object 
upon its impact against a surface.

Another experimental condition tested infant's

detection of temporal synchrony by controlling for temporal microstructure. This study was conducted by presenting infants in the first viewing of the preference phase with two films of the same objects side by side and with the soundtrack synchronized to only one of the films. During the second viewing the infants saw two films of the other object and heard its natural soundtrack again synchronized with only one film. Following the preference phase, there was a 30 second silent familiarization period where the two different events were presented side by side. Following the silent familiarization period, the search phase was conducted where brief presentations of each soundtrack were played out of synchrony with the motions of the two objects. Six-month-olds showed significant search to the sound specified film. For the second viewing of the preference phase both $41 / 2$ and 6-month olds looked significantly more to the sound synchronized film when the two compound objects were played but not when the two single objects were played. Since the two films were identical and differed only in their synchrony relation to the soundtrack infants must have detected the sound matched film on this basis. Therefore under some conditions both $41 / 2$ and 6-month-olds can detect audiovisual synchrony. The results indicated strong evidence 
for 6-month-olds but only minimal evidence for 4 1/2- montholds' detection of synchrony.

Overall these results lend support to previous research (Bahrick, 1983; Dodd, 1979; Spelke, 1981) that infants can detect temporal synchrony uniting a visible and audible display. The evidence was greatest for the 6-month-old group, however the $41 / 2$-month olds also demonstrated this capability under some conditions.

\section{Intermodal Learning}

Another major approach to the study of infants' perception has been to investigate the process of intermodal learning. One method involves familiarizing the infant to a specific object sound relation and then testing for auditory visual association by presenting the familiar and novel object side by side with either the familiar or novel sound. Using this method, Humphrey, Tees and Werker (1979) assessed 4-month-olds' ability to learn auditory visual correspondences when lights and sounds were in versus out of synchrony during familiarization. In the first experiment one group of infants was presented with temporally synchronized lights and sounds for the habituation trials and then were presented with asynchronous signals for the recovery trials. A second group of infants received the opposite sequence. Auditory and visual events were spatially congruent for both groups in the first experiment. The group 
receiving the synchronized sound and light for habituation showed significant habituation and recovery of looking time to the nonsynchronized pattern while the group receiving nonsynchronized presentations showed neither. These results suggest that infants in this study seemed to have been responding to the synchrony relationship between the light and sound and thus were able to demonstrate significant habituation and recovery. These results were replicated in a second experiment, when the locus of sound was 90 degrees right or left of the infant's midline. Overall these results suggest that spatial congruence is not a necessary condition for intermodal coordination if synchrony is present.

Lawson (1980) also used an intermodal learning method to study the learning of auditory visual relationships in 6month-olds by varying both spatial and temporal factors. Infants were familiarized with a moving object accompanied by a sound, followed by a test phase of two stationary objects, one novel and one familiar. On one test the objects were accompanied by the familiar sound and on the other by the novel sound. The experiments differed in terms of the spatial and temporal relations that were present during familiarization. In one experimental condition, the object moved periodically in synchrony with a sound emanating from it. When the familiar sound was played in the test phase infants looked longer at the object with which the sound had 
been paired than to the novel object. Thus 6-month-olds were able to match the object and sound when previous experience was with the spatially congruent and temporally synchronized pattern. In another experimental condition, the object moved in synchrony with the sound during familiarization but the sound was displaced 90 degrees to the left of the infant. Here the infants did not look preferentially at either object on the test. From these results it can be concluded that temporal synchrony between the sound and object is not sufficient for the infant to perceive a relationship. It is necessary for the auditory visual event to be spatially congruent and exhibit a synchronous temporal pattern for infants to make an association. These conclusions contrast with those presented by Humphrey, Tees and Werker (1979) who believed that synchrony between stimuli and not spatial congruence is the most important factor for infants to detect a relation between an auditory and a visual stimulus. Spelke (1981) tested whether 4-month-old infants who had learned an audiovisual relationship through synchrony would use this knowledge to guide subsequent visual search. In the initial phase of each of the 4 experiments, infants were presented with two different puppets that bounced at the same rate but with one film out of phase with the other. A sound synchronized to one puppet was played for a specified amount of time, followed by a different sound 
synchronized to the other puppet for the same period of time. Experiment 1 and 3 were followed by a search task in which the sound was out of synchrony with either puppet. Even though the infants did not look differentially in the preference phase, on the search task they looked first more often at the object that had been in synchrony with the sound even after the lateral positions of the objects were changed. In experiment 2 and 4 , the preference phase was followed by a transfer task, where the soundtrack and visual stimuli were presented successively; the soundtrack was first heard followed by the visual displays alone in silence. Infants now looked at the novel object, that is the object that had not been associated with the preceding sound. Infants looked to the novel object in this test as there was no spatial or temporal information present to unite the sight and sound of the object. These results suggest that witnessing an object moving in synchrony with a sound for a few minutes is enough to establish a link between them. Evidence for this relationship was stronger during the search task when the auditory and visual stimuli were present simultaneously than in the transfer task. Bahrick (1988) used an intermodal learning test to determine whether 3 -month-old infants are capable of uniting the audible and visible stimulation from a natural event. That is, infants were given the opportunity to view a film 
of a single large object as well as a film of a group of smaller objects colliding against a surface. Each film was accompanied by a soundtrack. The films and soundtracks were united by the synchrony between the motions and sounds of the object's impacts as well as the audio-visual information specifying the composition of the object, (whether it was a single large object or a group of smaller objects). Infants were trained in 4 familiarization conditions where the film and soundtrack pairings were either 1) appropriate and synchronous $(A / S), 2$ ) appropriate and nonsynchronous (A/N), 3) inappropriate and synchronous (I/S), or 4) inappropriate and nonsynchronous $(I / N)$. Therefore infants in the $(A / S)$ condition viewed the films along with their natural synchronized soundtrack, while those in the $(A / N)$ condition heard the natural soundtrack out of synchrony with the motions of the objects. Subjects in the (I/S) condition viewed the film of the single object moving in synchrony with the impact sounds of the group of smaller objects and the film of the group of marbles moving in synchrony with the impact sound of the single object. Finally those in the (I/N) condition viewed the film of the single object with nonsynchronous sounds of the group of marbles and the film of the group of marbles along with nonsynchronous sounds of the single marble. Then they were tested in an intermodal learning test in which films of the single object and the 
group of objects were presented side by side and were accompanied by soundtracks that were synchronous with the motions of both objects but appropriate to the composition of only one of the objects. Results indicated that the infants who were familiarized with the appropriate and synchronous film and soundtrack pairings looked significantly longer to the sound specified film during the test phase than the control group who received no training. Therefore, although all infants had an equal opportunity to associate the film to the soundtrack during training, only those infants in the $(\mathrm{A} / \mathrm{S})$ group demonstrated evidence of intermodal learning. The performance of infants who received inappropriate or nonsynchronous sounds was not significantly different from that of the control group and thus no evidence of learning was shown. The author reasoned that infants in this study learned the relationship between the audible and visible events on the basis of two types of invariant relations, synchrony and temporal microstructure, specifying the composition of the object. Synchrony in and of itself was not sufficient to promote learning in this study

One of the criticisms levied against Lawson (1980) is that learning may have occurred on the basis of association, that is, that a single object and a sound were always presented together during familiarization. However Bahrick's 
(1988) intermodal learning method provided a control for learning occurring on this basis. That is, although infants in all four familiarization conditions had an equal opportunity to detect a co-occurring visual event with a soundtrack, only those who were familiarized with the appropriate and synchronous event displayed evidence of learning the relationship between the visual event and the sound. Therefore learning in this study did not occur on the basis of co-occurrence.

Overall these learning studies suggest that infants learn to relate objects with sounds on the basis of temporal synchrony (Humphrey, Tees and Werker, 1979; Lawson, 1980; Spelke, 1981; Bahrick,1988) as well as temporal microstructure which specifies the composition of an object (Bahrick, 1988) and simple audiovisual co-occurrence (Lawson,1980). But they do not relate objects to sounds when the soundtracks are either nonsynchronous or inappropriate to the composition of the object (Bahrick,1988). Perception of Object Motion

The above studies have firmly established that infants by the age of 6 months are able to detect through synchrony the unity of the optic and acoustic stimulation in the world around them. These findings are also consistent with a developmental sequence of temporal feature differentiation proposed by Lewkowicz (in press) where the detection of 
temporal synchrony is proposed to emerge before the detection of duration, rate and rhythm. He also believes that the differentiation of each temporal feature is dependent on the differentiation of the previous one. However it is not clear from these studies how global or specific the detection of synchrony is. Spelke, Born and Chu (1983) conducted their research in an attempt to shed more light on this issue. Their research examined the nature of the changes in a visual display that were synchronized with a sound in order for infants to detect such a relationship. Three possibilities were examined namely, whether infants detected synchrony when the sound was synchronized with the object's impact, or to the object's change in direction of motion, or to the object's arrival at a specific spatial location. The design utilized in this experiment was similar to Spelke (1979) with preference trials followed by a series of search trials, however the visual displays involved two real puppets rather than films and their rates of movement were slower. The first experiment was conducted to determine if infants could detect synchrony when a sound was synchronized with an impact, a change in direction of motion and an arrival at a specific spatial location. Infants viewed two puppets moving up and down hitting the stage in a given spot. Both puppets moved at the same rate of motion but out of phase with each other. During the first trial of 
the preference episode one sound was synchronized with one of the objects and during the second trial the other sound was synchronized to the other object. Infants looked longer at the visually synchronized object in the preference episode and directed more attention to the sound matched object in the search phase. From these results it can be concluded that infants detected an audiovisual relation. They may have detected a relation between the sound and the object's impact, the change in direction of motion, or the arrival at a specific location. Subsequent experiments attempted to narrow down which of these correspondences were detected by the infants.

The purpose of experiment 2 was to determine if infants could detect a relationship between a sound and a synchronized pause and change in trajectory of an object even when no visual impact accompanied the sound. In this experiment the sounds were synchronized with the object's pause in midair and change in direction of motion. The object always paused at the same point in space. The motions of the other puppet were not systematically related to the sound. It paused and changed trajectory when no impact sound was heard. The infant's behavior in both the preference and search episode was essentially the same as their behavior in the first experiment. The authors concluded that infants relate percussive impact sounds to any abrupt change in the 
movement of an object. Although the author's conclusion is valid it is also possible that the impact sound could have been related to the pause only, the change in direction of motion or spatial position or any combination of these factors.

Experiment 3 investigated the importance of visual impact versus change in trajectory for detecting an auditory visual relationship between an object's motions and sounds. Would infants look more to an object that pauses and impacts a surface than to one that pauses and changes its trajectory in midair when a percussive sound occurs? Here two puppets were moved in synchrony with one another. One object paused on contact with the surface while the other paused in midair. The sound was synchronized to one puppet's impact and to the other's pauses in midair. Infants showed no differential looking in the preference or search phases. Therefore, this study provided evidence that infants do not relate a percussive sound more to an impact with a surface than to a pause in midair. However this changes in adulthood as research demonstrated that adults perceive sounds to be related to impacts rather than a change in trajectory.

Experiment 4 investigated whether infants were sensitive to the relation between an impact sound and the spatial position of an object when it moves continuously. The procedure was the same as experiment 1 except that the 
two objects were moved at a constant speed in circles and at a slightly different rate of motion so that the relative positions of the objects changed continuously as the objects were moved. A percussive sound was heard each time one of the objects reached a specified spatial position on its circle. The sound was not predictably related to the position of the other object. There was no tendency for infants to look longer at the object whose sound was synchronized with a particular spatial position. Infants appear not to relate a sound to spatial position when there is no pause or change in trajectory present. This experiment demonstrated no evidence for detection of a relationship between the spatial position of an object and an impact sound.

Experiment 5 looked at the relationship between an impact sound and change in direction of motion and pause when spatial position of change varies. Here the objects moved clockwise 360 degrees and paused, then moved counter clockwise 380 degrees. The objects' motions were out of synchrony with each other and an impact sound occurred whenever one object changed direction. Position of change varied throughout the experiment. No significant effects were found for the preference episodes. However there was a significant effect during the search episode. Infants looked first to the sound synchronized object. Also the sound 
synchronized object was viewed with a shorter latency and for a longer duration. Infants seem to detect an audiovisual relation when the sounds are synchronized with changes in direction of motion even though spatial position of the object at change varies.

A final study with adults suggests that development may involve increasing specificity of response as the adults showed strong results only for the visual displays involving impact with the ground.

Overall these results indicated that infants perceived correspondences between an object and a sound when there is a pause and change in trajectory of an object. Although there was a pause present in all of the experimental conditions, with the exception of experiment 4, the authors never addressed this issue. Perhaps the pause contributed to the infant's detection of synchrony when there was a change in the movement of an object. Therefore the present study will seek to take these results a step further by examining whether matching will generalize to the synchrony between sounds of impact and pauses in an object moving in a continuous circular motion and with no visible surface present. Infants at $21 / 2,4$ and 6 months of age were tested to determine whether they could relate an impact sound to an object which pauses only and also to determine whether there are differences in the detection of audiovisual 
correspondences across age. More specifically, will older infants like adults respond to a sound-object relationship when sounds are synchronized with an impact? The research was designed with specific controls for the spatial location of the pause to rule out spatial location as a contributing factor in the detection of correspondences.

Although this paper does not set out to prove or to refute any of the major theories of perception, the hypotheses are consistent with Gibson's (1969) invariant detection view as it demonstrates that infants from early on in life are capable of abstracting meaningful properties of objects through the detection of invariants. On the other hand, this evidence of early detection of intermodal knowledge weakens the integration hypothesis which suggests that sensory systems function independently during early development and become increasingly integrated with age (Birch \& Lefford, 1963). That is, only through time and experience can infants detect an audiovisual correspondence between an object and a sound. Piaget's (1952) view also suggests that intermodal coordination at an early age is not possible as perception and knowledge are dependent on the child acting on his or her environment and at this age motor behavior is not yet well developed. 


\section{Chapter 3 \\ Experimental Design}

\section{Experiment 1}

Prior research has shown that infants match sounds with a synchronized change in trajectory of motion, even when no impact of the object with a surface is visible. However they do not relate an impact sound to the spatial position of an object when there is no pause or change in trajectory present (Spelke, Born \& Chu, 1983). The following study examined whether infants would match an impact sound to a continuously moving object that paused intermittently in synchrony with an impact sound. In this experiment no impact, no trajectory change or common space location were present when the impact sound occurred.

A modified version of Spelke's (1979) intermodal preference and search method was used. During the preference phases, subjects saw two films side by side along with a soundtrack to one of them coming from a centrally placed speaker. The sound was centrally located to prevent infants from localizing the sound to either the right or left side. During the first viewing of the preference phase the two films were projected for 60 seconds along with the soundtrack to one of them. In the second viewing of the preference phase, the video films were again presented on the same side for an additional 60 seconds however this time 
the soundtrack to the other previously silent film was heard. It was predicted that infants would look more to the visually synchronized object which paused when the sound occurred if they related the sound to the object's pause even though no trajectory change was present. Next a search phase was conducted which was similar to that of Bahrick (1983) and was included to assess what was learned during the preference phase. During the search phase both video displays were viewed side by side for about 3 minutes. For a given infant, the displays were projected on the same side as in the preference phase. The measure of interest was the infant's first look upon hearing the soundtrack. It was predicted that infants would look first more often to the display whose pauses were previously synchronized with the sound during the preference phase. This prediction is based on the assumption that infants learned the correct object sound pairing during the preference phase.

\section{Method}

Subjects: Seven 4-month-olds ( 3 males and 4 females, with a mean age of 121.3 days, $\underline{S D}=12.2$ ) and 116 -montholds ( 4 males and 7 females, with a mean age of 187.6 days, $\underline{S D}=7.2$ days) participated. Ten additional infants ( 86 month-olds and 24 -month-olds) were tested and eliminated from the study because of experimenter error ( $\underline{N}=5)$ or not producing enough usable trials on the search phase $(\underline{N}=5)$. 
All were healthy full term infants recruited through the local birth records.

Stimulus Materials: Video films of a red wooden square decorated with white circles and a blue triangle with yellow stripes served as the stimuli. Each object was depicted moving in a circular path by rotating a wooden wheel. This was accomplished by projecting the handle of each wheel through a circular slot in a black posterboard while the rest of the wheel was hidden from view. Each object was then attached to the handle of the wheel and two separate experimenters turned each wheel from behind the posterboard so that they were out of view. This was done so that the objects would appear to move in synchrony with each other. Color video films were made of the objects moving in a circular trajectory, pausing approximately 20 times per minute. A sound was synchronized with each pause and here there was no change in trajectory. Two types of percussive sounds were recorded in synchrony with the pauses. A programmable rhythm machine (E-mu Systems, Inc.) was used to produce these sounds. One of the sounds produced was a dull thud sound of a snare drum (low pitch) while the other was a single discrete tone of a clave (high pitch). Pauses occurred according to a predetermined pattern, which consisted of a 23 beat sequence and with the sound occurring on the fourth, ninth, twelfth, eighteenth and twenty first 
beats. This pattern was selected so that the object would pause at different points along its circular trajectory. Synchrony between each pause and the sound was achieved by having a separate experimenter move each wheel according to the above pattern. That is, both experimenters stopped and started the wheel in unison with the sound from the rhythm machine. The same objects were used for each of four experiments. Finally, as a double check to determine if the objects could be matched to the sounds, an adult survey was conducted. A Panasonic Digital AV Mixer WJ-MX12 was used to superimpose one film on the other so that both objects were visible on one television screen while only one sound was played. The objects were not presented side by side but were displayed on one screen so that the task would be more difficult for the adult viewers, also the procedure used would focus their attention on the task at hand. It was believed that presenting the objects on two screens would be too obvious for these viewers. Judges were asked to determine which object was synchronized with the soundtrack. All judges $(n=5)$ selected the correct object and their selection was based on the fact that the object paused when the sound occurred.

Apparatus: Infants were seated in an infant seat enclosed by curtains. They sat approximately 20-inches away from two 19-inch color monitors ( Panasonic AK-1090). The monitors 
were placed approximately 5 inches apart and a strip of colored Christmas tree lights was placed in between the two monitors to center the infant's attention between trials. The displays were presented with a Panasonic VHS NV-A500 edit controller connected to two Panasonic video decks (NV8500 and AG-6300). The soundtracks from both films were presented from a centrally located speaker. The infant's visual fixations to the displays were recorded by an 8channel Rustrak strip-chart recorder.

Procedure: When the infant arrived at the laboratory, the purpose of the experiment was explained to the parents. The parents were then asked to sign a consent form and were permitted to enter the testing room with the infant. During the preference phase, infants viewed video displays of a red and white square and a blue and yellow triangle side by side and moving in a circular path. One of two sounds was played and was synchronized to the pauses of one of the objects while the other object's pauses were out of synchrony with the sound. During preference phase 2, the other sound was played in synchrony with the pauses of the previously silent object and was out of synchrony with the other object's pauses. The order of the soundtrack presentation as well as the lateral positions of the films were counterbalanced across all subjects in each age group. Three 4-month-old infants saw the blue object on the left, one of these 
infants heard the sound of the snare drum first and the other two infants heard the sound of the clave first. Four of the infants saw the red object on the left, two of these infants heard the sound of the clave first and the other two heard the sound of the snare drum first. In the 6-month-old group, six of the infants saw the blue object on the left, three heard the sound of the snare drum first and the other three heard the clave first while the other five 6-montholds saw the red object on the left, three heard the snare drum first and the other two heard the clave first. The preference session was always followed by a search phase. During the search phase both video displays were presented side by side again moving as before and were accompanied by 3-4 seconds of intermittent presentations of each of the two synchronized soundtracks. The lateral position of the films were constant across the preference and search phase for a given infant. The measure of interest was the infant's first look upon hearing the soundtrack. Because of this the trial began whenever the infant fixated the center screen. The infant's attention was attracted to the center screen either by flashing the Christmas tree lights on and off, by moving the mechanical toy dog, or only if the first two failed by the experimenter snapping her fingers in between the center of the two monitors. The soundtrack was played only when the infant fixated the center screen and therefore the subject's 
visual behavior determined the onset of each trial. The number of trials each infant received varied from as few as five up to nineteen usable trials, with a criterion of five usable trials being preset in order for the data to be included in this part of the experiment.

Trained observers who were blind to the lateral positions of the films monitored the infant's visual fixation from small apertures cut into the curtains to the right and left of the television monitors. The observers depressed one of two buttons depending on whether the infant fixated the right hand display or the left hand display. A second observer monitored the subject's fixations for $37 \%$ of the sample for the purpose of calculating interobserver reliability. Interobserver reliability for the trial session and search phase were .97 and .94 respectively. This is a Pearson product-moment correlation between the proportions of total looking time the infant spent fixating the soundmatched film as derived from observations of the primary and secondary observers.

\section{Results}

Overall, the 4-month-old infants looked to the films about 103s out of the total $120 \mathrm{~s}$ ( $86 \%$ of the time), and the 6-month-old infants looked $97 \mathrm{~s}$ ( $81 \%$ of the time). Both age groups showed a decline in total looking time to the video displays across preference phases. Four-month-olds looked an 
average of 53.4s during preference phase 1 and an average of $49.6 \mathrm{~s}$ during preference phase $2(\underline{t}(6)=1.02, \underline{p}>.05)$. Sixmonth-olds looked an average of $53.9 \mathrm{~s}$ on preference phase 1 as compared to 49.9 secs on preference phase 2 (t $(10)=$ $1.79, \underline{p}>.05)$. Although infants looked less across the two preference phases this was not statistically greater than chance thus indicating that infants were not significantly bored or fatigued at the end of the task.

Visual fixations were expressed in terms of the proportion of total looking time (PTLT) the infants spent fixating the sound matched film out of the total time they spent looking to both films. This was calculated separately for each preference phase and averaged across the two preference trials to obtain a grand mean proportion for each subject. The mean proportion of first looks directed toward the sound-specified film was calculated for the search phase. This was obtained by dividing the number of trials on which the infant looked first to the sound-specified film by the total number of trials produced.

A oneway analysis of variance was conducted to assess whether there were differences in looking to the soundmatched presentation between the two age groups on preference phase 1 , preference phase 2 and the average of both preference phases. However no differences were found between the two age groups on preference phase $1, E(1,17)$ 
$=3.00, \underline{\mathrm{p}}>.05$, preference phase $2, \underline{\mathrm{F}}(1,17)=1.92, \underline{\mathrm{p}}$ $>.05$ or the average of both preference phases, $\underline{F}(1,17)=$ $.070, \underline{p}>.05$.

Single sample t-tests comparing the mean looking preferences for the sound-specified films against a chance value of .5 were performed on each preference phase separately and the average of the two preference trials for each of the two age groups. The mean PTLTs to the sound matched film for each age group are shown in Table 1 . When the two preference phases were averaged together 6-montholds spent a significantly greater proportion of the time viewing the mismatched presentation, that is the film which did not pause in synchrony with the sound, $\underline{t}(10)=-2.41, \underline{p}$ $<.05$. On the other hand, 4-month-olds demonstrated no significant looking to the sound-matched films, $\underline{t}(6)=-$ $1.63, \underline{p}>.05$.

A repeated measure analysis of variance was performed to see whether infants displayed any trends over time in looking to the sound matched film during preference phase 1 or preference phase 2 for each age group. Results for the 6month-olds indicated there were no trends over time in looking across preference phase 1 and preference phase 2, F $(1,10)=1.14, \underline{p}>.05$. The findings for the 4-month-olds were similar to those of the 6 -month-olds on this measure, $\underline{F}$ $(1,6)=1.37, \underline{p}>.05$. Each trial was divided into thirty 
20-s intervals to see if infants at either age group displayed trends in looking to the sound-matched object across trials. Results for the 6-month-olds indicated there were no differences across the 20-s intervals, $\underline{F}(2,20)=$ $.53, \underline{p}>.05$. However upon closer analysis of preference phase one it appears that their performance seemed to have worsened by the end of the trial ( $\underline{M}=.58, \underline{M}=.54, \underline{M}=.47)$ thus indicating that the task might have been too easy for these infants, that is, they quickly moved from looking at the sound-matching film to the mismatching one. Single sample ttests comparing these means against a chance value of .5 yielded results which were not statistically different from chance.

A repeated measures analysis conducted on the 20-s intervals of both preference phases for the 4-month-olds also revealed no differences, $\underline{F}(2,12)=1.14, \underline{p}>.05$. However further analysis indicated that the 4-month-olds displayed a trend towards significance on viewing period 2 $(\underline{M}=.64, \underline{M}=.49, \underline{M}=.56)$ and the search phase $(\underline{M}=.56)$. This may mean that these infants needed more time to understand what was required of them. Single sample t-tests performed on these means indicated that they were not significantly different from chance responding.

Results of the search phase yielded no significant results for either age group, although the 6-month-old group 
displayed a tendency to look towards the mismatched object while the 4-month-olds had a tendency to look towards the matched object.

Further analyses were conducted to determine whether infants displayed any side or object preferences. Results indicated that the 6-month-old infants showed a significant preference for the red display $(\underline{t}(10)=3.86, \underline{p}<.01)$. A difference score was calculated to examine the PTLT to the red object when the red object was sound matched minus the PTLT to the red object when the object was sound mismatched for this age group revealed that even when the object preference was partialled out the 6 -month-old infants still looked to the mismatched object $(\underline{M}=-.09, \underline{t}(10)=-2.25, \underline{p}$ $=.05)$.

\section{Discussion}

Six-month-olds in this study demonstrated a significant looking preference for the object which paused out of synchrony with the sound and this was displayed when both preference trials were averaged together. Four-montholds demonstrated no preference. Similar results of infants displaying a preference for the non-synchronous event have been documented by Bahrick (1983). Her results indicated that 4 1/2-month-old infants were able to match films and soundtracks on the basis of synchrony and on the basis of elasticity or rigidity of substance of the object. However 
like in the present study, they showed detection of synchrony by a significant preference for the nonsynchronous object. It is not clear why infants sometimes show evidence of matching by looking significantly more to a nonsynchronous event. However it is possible that infants may have looked to the mismatched event in this experiment as the task was too easy and they became bored, or perhaps by 6 months of age, infants are familiar with the idea of synchronous sights and therefore found the non-synchronous presentation more interesting or novel to attend to. On the other hand, other research has shown that infants by the age of 4 months are able to match impact sounds to objects (Spelke, 1979, \& 1981) therefore it was hypothesized that 4month-olds were probably unable to match the sound to the synchronous event in this experiment as the trials may have been too long. Also, when this procedure was used in a prior study it was found that infants tend to demonstrate an acquired position response. That is, if they had been looking at a display on the left side they began the next trial with a dominant tendency to look at that side even though stimulus conditions had been changed. Also, perhaps infants were unable to match the sights and sounds as the sounds presented were impact sounds and the objects were not showing an impact motion. Therefore in the next experiment the procedure was changed by replacing the 2 long trials 
with shorter preference trials. This procedure had been used successfully by Bahrick (1988) with infants as young as 3 months of age.

\section{Experiment 2}

The above method may have been unsuccessful in eliciting the object-sound matching for the 4-month-old group as the trials may have been too long. Therefore for the next two studies the two long trials were replaced with a number of shorter preference trials. The advantage of this method is that the use of shorter trials would serve to orient the infant back to the center of the screen and then force the infant to once again choose a film when the sound came on. Also it would allow us to see whether the 6 -montholds would continue to show the mismatching effect or whether by making the task more interesting and variable the 6-month-olds would look significantly more to the synchronous event before turning to look at the nonsynchronous one. Therefore Experiment 2 was conducted to attempt to get the 6 -month-olds to match the object's pauses with the sounds. As this experiment was intended to be a pilot for this method, only 6 -month-olds were tested in this procedure. 


\section{Method}

Subjects: Sixteen 6-month-old infants, 12 males and 4 females participated. Their mean age was 185.4 days, $\underline{\mathrm{SD}}=$ 6.20 days. Five additional infants were tested and excluded from the study because of equipment failure ( $\underline{N}=1)$, experimenter error $(\underline{N}=2)$ or not producing enough usable trials on the search phase $(\underline{N}=2)$.

Apparatus \& Procedure: The apparatus was identical to that used in Experiment 1. However infants now viewed $1215 \mathrm{~s}$ trials comprised of two blocks of six trials. Each trial depicted video displays of the red and white object and the blue and yellow triangle side by side, and moving in a circular path. One of two sounds was played for the first block of six trials and was synchronized to the pauses of one of the objects while the other object's pauses were out of synchrony with the sound. The second sound was then played for the second block of six trials in synchrony with the pauses of the previously silent object and were out of synchrony with the other object's pauses. Thus, although the sound remained constant for each block of trials the lateral positions of the displays varied randomly across trials. It was predicted that infants would look more to the visually synchronized object which paused when the sound occurred if they related the sound to the object's pause even though no trajectory change was present. During the search phase both 
video displays were presented side by side again moving as before and were accompanied by 3-4 seconds of intermittent presentations of each of the two synchronized soundtracks. It was predicted that infants would look first more often to the display whose pauses were previously synchronized with the sound during the trial session. This prediction was based on the assumption that infants learned the correct object sound pairing during the trial session.

\section{Results}

Overall, the 6-month-old infants in this experiment fixated the films approximately $123 \mathrm{~s}$ out of the total 180s, or $69 \%$ of the time. Visual fixations were expressed as the proportion of total looking time (PTLT) the infant spent looking to the sound specified film out of the total time they spent looking to both films on each test trial. These proportions were then averaged across each trial block (Trials 1 to 6 and Trials 7 to 12 ) to yield two mean proportions for each infant. A grand mean PTLT was also obtained for each infant by averaging the two means across trial blocks. The mean proportion of first looks directed toward the sound specified film was calculated for the search phase. This was obtained by dividing the number of trials on which the infant looked first to the soundspecified film by the total number of trials produced.

In order to test the main hypothesis, single sample $\underline{t}-$ 
tests were performed on the PTLT to the sound matched film for each block of trials. This was done to compare the mean looking preferences for the sound-specified film against a chance value of .5. Table 2 indicates the PTLT to the sound matched film for this experiment. Results indicated no evidence of matching or mismatching to the sound specified film on block 1 and 2 together, $\underline{t}(15)=-1.45$, ㄹ $>.05$, or block 1, $\underline{\mathrm{t}}(15)=-1.31, \underline{\mathrm{p}}>.05$, or block $2, \underline{\mathrm{t}}(15)=-.44$, $\underline{p}>.05$. Single sample t-tests were also conducted on the proportion of first looks directed towards the sound specified film during search trials. This was done to determine if infants looked first more often to the sound film. Results showed infants did not search reliably to the sound matched object in this experiment, $\underline{t}(15)=-.65, \underline{p}>$ .05 .

\section{Discussion}

Results of this experiment indicated that infants at 6 months of age are unable to match a sound with an object that pauses under the present conditions. Although these results show that infants once again had a tendency to look more at the nonsynchronous display this was not statistically significant. This method may have been unsuccessful because the procedure was too complicated. Infants may have been unable to learn the correct soundobject relationship because they were unable to predict the 
side on which each object would appear. The unpredictability of the side of the films may have made the task too

difficult. Since we were unable to replicate the mismatching effect which was found in experiment 1 with the 6-month-old infants this procedure was simplified further for the next experiment and was conducted on both 4 - and 6-month-old infants.

\section{Experiment 3}

The procedure was simplified for this experiment by keeping the films on the same side rather than varying the lateral positions of the displays. This procedure should be easier as infants were given an opportunity to learn on which side each object appeared and then could anticipate where to look when each sound was heard.

\section{Method}

Subjects: Twenty-four 6-month-olds ( 14 males and 10 females, with a mean age of 186.7 days, $\underline{S D}=8.0$ days) and 16 4 -month-olds ( 8 males and 8 females with a mean age of 117.2 days, $\underline{S D}=7.9$ days) participated. Nine additional infants (4 4-month-olds and 5 6-month-olds) were tested and eliminated from the study because of equipment failure $(\underline{N}=4)$, experiment error $(\underline{N}=2)$ or not producing enough usable trials on the search phase $(\underline{N}=3)$.

Apparatus \& Procedure: The apparatus and procedures were the same as those used in Experiment 2 except for the 
following: Half the infants in each age group received the films on the same side for all twelve trials. They were accompanied by one of two soundtracks presented in a semirandom order. Each infant received three presentations of each sound in each block of six trials and a given soundtrack was not played more than twice in succession. The selected soundtrack order was played for the first block of six trials and repeated for the second block of six trials. The other half of infants in each age group had the position of the films reversed for the second trial block but received the same soundtrack order across block 1 and block 2. The lateral positions of the objects were counterbalanced across subjects with respect to subjects who received the reversal versus those who did not.

\section{Results}

Overall, the 4-month-old infants looked to the films about $133 \mathrm{~s}$ out of the total $180 \mathrm{~s}$ ( $74 \%$ of the time), the $6-$ month-old infants looked 129s (72\% of the time). Both age groups demonstrated a decline in total looking to the displays during Trials 7 to 12 as compared to Trials 1 to 6 . Four-month-olds looked an average of $69.6 \mathrm{~s}$ or $77 \%$ of the time during Trials 1 to 6 and 63.9 or $71 \%$ during Trials 7 to 12, $\underline{t}(24)=2.47, \underline{p}<.05$. Six month-olds looked an average of $69.7 \mathrm{~s}$ or $77 \%$ during Trials 1 to 6 and $59.5 \mathrm{~s}$ or $66 \%$ on Trials 7 to 12 , $\underline{t}(35)=4.48, \underline{p}<.05$. Thus there was a 
significant decrease in looking between Block 1 and Block 2 for both age groups. These results are similar to those of Experiment 1 and indicate that subjects were less interested in the film and were probably fatigued by the end of the session.

In order to assess whether subjects' looking preferences differed across the two age groups (4 months, 6 months) a two way repeated measures analyses of variance was conducted on the PTLT across each trial block for 4 and 6month old infants. The results indicated no significant differences between the two age groups for an interaction of age by block, $\underline{F}(1,38)=1.20, \underline{p}>.05$ or for a main effect of age, $\underline{F}(1,38)=1.65, \underline{p}>.05$ or for a main effect of block, $\underline{F}$ $(1,38)=.06, \underline{p}>.05$.

A three way analyses of variance was conducted on the proportion of first looks directed towards the soundspecified film during the search trials and across each block of trials for both 4-month-old and 6-month-old infants. Results indicated no significant first looks on block 1, $\underline{F}(1,38)=3.81, p>.05$, block $2, \underline{F}(1,38)=2.75$, $p>.05$ and across search trials, $\underline{F}(1,38)=.96, p>.05$. In order to test the main hypothesis, single sample $\underline{t}-$ tests were conducted separately for each age group and for each block of trials on the PTLT to the sound matched films. This was done to compare the mean looking preferences for 
the sound-specified films against a chance value of .5. The mean proportion of first looks directed towards the soundspecified film was calculated for the preference trials. This measure was obtained by dividing the number of preference trials on which the infant looked first, after the onset of the sound to the synchronized object by the total number of preference trials presented. This dependent variable indicated whether infants looked first more often at the synchronized object immediately after the sound was played. This measure was not calculated for experiment 2 because the lateral positions of the displays varied randomly across the experiment, making it impossible for infants to predict the side on which each display would appear. Table 3 provides PTLTs and first looks to the soundmatched film for this experiment. The 4-month-old infants showed significant evidence of matching and this was only evident on the second block of six trials, $\underline{t}(15)=2.19, \underline{p}<$ .05. Single sample t-tests were also conducted on the proportion of first looks directed towards the sound specified film during the search trials and across each block of trials. The 4-month-old infants looked first to the object which paused with the sound across both trial blocks together and block 2 alone, $\underline{t}(15)=4.61, \underline{p}<.01, \underline{t}(15)=$ $3.06, \underline{p}<.01$ and with marginally significant first looks on block 1, $p=.06$. The significant first looks which were 
found in this experiment support the hypothesis that the task of Experiment 1 was probably boring for these infants. Four-month-old infants may not have been motivated to perform the task in Experiment 1 as the trials may have been too long. Each infant in experiment 1 was presented with the films for two 60 s trials. The use of shorter trials as in experiment 3 , served to orient the infant back to the center at the beginning of each trial. Also the reversal of the lateral position of the displays after the first trial block served to control for the issue of captured attention. Sixmonth-olds only demonstrated significant first looks during search trials, $\underline{t}(23)=-2.20, \underline{p}<.05$. However, here the infants looked more to the mismatched object. Similar to experiment 1, 6-month-olds once again found the task too easy. In experiment 1 , infants demonstrated their ability to detect synchrony by looking significantly more to the object which paused out of synchrony with the sound and this was detected when both preference trials were averaged together. In experiment 3 , this ability was once again demonstrated by looking significantly more to the mismatched object on the search phase. These infants demonstrated no preference on experiment 2 as the task may have been too confusing. In summary, the 4-month-old infants were able to match the sound with the object which paused and this was demonstrated on block 2 and also on the first look proportions across 
block 1 and 2 together and block 2 alone, whereas the 6month-olds showed significant first looks during search trials and this was to the mismatched object.

A two way analyses of variance was conducted on the PTLT for block 1 and 2 together to see if there were differences between the 4-and 6-month-olds infants who received the film on the same side versus those who did not. Results indicated no main effects for age group, $E(1,39)=$ $1.84, \underline{\mathrm{p}}>.05$, no main effect for the reversal, $\underline{\mathrm{F}}(1,39)=$ $.003, \underline{p}>.05$ or for the interaction of age group and reversal, $\underline{F}(1,39)=.07, \underline{p}>.05$. A three way repeated measures analyses of the variance was also performed on PTLT for block 1 and block 2 with age group and reversal as factors. Results revealed no significant main effects for age group, $\underline{E}(1,36)=1.61, \underline{p}>.05$ or reversal, $\underline{F}(1,36)$ $=.00, \underline{\mathrm{p}}>.05$ or no significant three way interaction of age group by block by reversal, $\underline{F}(1,36)=.03$, ㅁ $>.05$. A similar three way repeated measures analyses of the variance was performed on first looks across block 1 and 2 with age and reversal also included as factors. There was a significant main effect of age across both trial blocks 1 and $2, \underline{F}(1,36)=6.49, \underline{p}=.02$. However no main effect was found for the reversal $\underline{F}(1,36)=.02, \underline{p}>.05$. The three way interaction of age by reversal by block was also not statistically significant, $\underline{F}(1,36)=.13, \underline{p}>.05$. 
Single sample t-tests were further conducted to determine whether subjects who had received the films on the same side within each age group for all twelve trials did better than those who had the position of the films reversed after the first block. Results indicated no significant differences on PTLT to the sound matched films for 4-monthold. However, t-tests against .5 revealed that infants who received the films on the same side directed significantly more first looks to the sound-matched object than those who had the films reversed after the first block of trials (block 1 and 2 together and block 1 alone) $\underline{t}(7)=4.74, \underline{p}<$ $.01, \underline{t}(7)=2.65, \underline{p}<.05$ versus $\underline{t}(7)=2.29, \underline{p}>.05$ for block 1 and 2 together and $\underline{t}(7)=.55, \underline{p}>.05$ on block 1 . The means for these two groups are displayed in table 4 . This indicated that subjects who received the films on the same side for all twelve trials carried the matching effect. There were also no significant differences on PTLT or first looks for 6-month-olds who had the position of the films reversed after the first block of trials versus those who had the films on the same side for all twelve trials. However subjects who had the films reversed after the first block looked significantly more to the mismatched object on the search phase, $(\underline{t}(15)=-2.20, \underline{p}<.05)$ than those who had no reversal $(\underline{t}(15)=-0.68, \underline{p}>.05)$. One reason which may account for this finding in the 6-month-old group is 
that there were twice as many infants in the reversal condition versus those in the no reversal condition, $(\mathrm{N}=16$ versus $\mathrm{N}=8$ ).

A two way mixed ANOVA age by trial was conducted across all trials for 4 - and 6-month-olds to determine if subject's performance improved across trials. Results yielded no significant interactions for age by trial, $\underline{\mathrm{F}}(11,38)=1.20$, $\underline{p}>.05$ or main effect for trials $\underline{F}(11,38)=.86, \underline{p}>.05$ or age group, $\underline{E}(1,38)=1.98, \underline{p}>.05$.

Single sample t-tests revealed that 4 -month-olds displayed no significant side or film preferences. However 6-month-olds demonstrated significant looking preferences to the red object, $\underline{t}(23)=2.23, \underline{p}<.05$.

\section{Comparison Across Experiments}

In order to determine whether there were differences in looking preferences between Experiment 2 and Experiment 3 a one way analyses of variance was conducted on grand mean PTLT to the sound matched film for the 4-month-old and the two 6-month-old groups. Results revealed no significant differences between the three groups, $\underline{F}(2,53)=1.72$, $\underline{p}>$ .05. There was also no significant difference when the 4month-old group was compared to the two 6-month-old groups collapsed, $\underline{F}(1,54)=.3 .30, \underline{p}>.05$. A comparison of the search phase also yielded no significant differences, F $(1,54)=.53, \underline{p}>.05$. 
Further analyses were conducted to determine whether infants at either age or across the two experiments showed any preferences for the red or blue object or the right-or left-hand displays regardless of the sound presentation. Results indicated that the 6-month-old infants in both experiment $1, \underline{t}(15)=2.76, \underline{p}<.05$ and experiment $2, \underline{t}$ $(23)=2.23, \underline{p}<.05$, showed a significant preference in looking to the red display.

\section{Discussion}

Results of this experiment indicate that both 4-monthold and 6-month-old infants are able to detect temporal synchrony between sounds of impact and pauses in the movement of an object. Four-month-olds demonstrated detection of this relationship by looking significantly more to the synchronous display during block 2, whereas by 6months of age it appears that infants no longer use this sensitivity to guide visual selectivity as evidenced by the results of the search phase. They prefer to look to the mismatching display. Therefore since we know that infants by 4-months of age can relate a sound with a synchronous pause in continuous motion we wanted to see if this ability was present at a younger age. Therefore the following experiment was conducted with 2 1/2-month-old infants. This experiment would provide us with an interesting developmental trend of infants' ability to relate a sound to a synchronous pause in 
continuous motion.

\section{Experiment 4}

This study replicated experiment 3 with 2-1/2-month-old infants. Here we wanted to test if the ability to match impact sounds with an object's pauses was present by $2-1 / 2$ months of age.

\section{Method}

Subjects: Sixteen $2-1 / 2$ month olds $(8$ males and 8 females, with a mean age of 86.6 days, $\underline{S D}=6.3$ days) participated. One additional infant was tested and eliminated from the study because of not producing enough usable trials.

Apparatus \& Procedure: The apparatus was identical to that used in the preceding experiments. However, in this experiment the infants received no search phase. It was hypothesized that the search phase might be too difficult for these young infants as this task required greater attentional mobility and motor coordination. Instead they were given an additional block of six trials. In order for infants to be included in this study they needed to complete 10 out of the 18 trials. Half the infants in each group received the films on the same side for all eighteen trials while the other half had the position of the films reversed for the second and again for the third block of trials. The lateral positions of the objects were counterbalanced across subjects and the two soundtracks were presented in a semi- 
random order.

\section{Results}

Single sample $\underline{t}$-tests were conducted separately for each block of trials on the PTLT to the sound matched films as well as on first look proportions. Table 5 provides PTLTs and first looks for this experiment. Results revealed no significant looking preferences, $(\underline{t}(15)=-.80, \underline{p}>.05)$, nor significant first looks, $(\underline{t}(15)=.48, \underline{p}>.05)$, to the sound matched display. However, first look proportions do appear to be leaning in the direction of matching. Single sample t-tests were also conducted for infants who had the films reversed after each block of trials $(\underline{t}(7)=-.92, \underline{p}>$ .05 ) versus those who received the films on the same side $(\underline{t}(7)=.50, \underline{p}>.05$ throughout the test yielded no significant differences. A repeated measures analysis of variance revealed no effects of trial block for either the PTLT, $\underline{F}(2,30)=.46, \underline{p}>.05$ or first look measure, $\underline{F}(2,30)=.78, \underline{p}>.05$. Further analyses were conducted to determine whether infants in this experiment showed any preference for the red or blue object or the right-or-left hand displays regardless of the sound presentation. Results revealed these infants looked significantly more to the right hand display, $\underline{t}(15)=-2.07, \underline{p}=.05$ irrespective of sound. 


\section{Comparison Across Experiments}

In order to assess if there were any differences in looking preferences between the three age groups $(21 / 2,4$, 6) and across those subjects who had the position of the films reversed versus those who had the films on the same side an analysis of variance was conducted on the looking proportions for block 1 and 2 together, leaving out block 3 for the 2 1/2-month-old infants. Results indicated no significant differences between those subjects who had the position of the films reversed after the first trial block versus those who did not. There were no significant main effect of age group, $\underline{F}(2,55)=2.02, \underline{p}>.05$, or reversal, $\underline{F}(1,55)=1.85, \underline{p}>.05$ or the interaction of age group by reversal, $\underline{F}(2,55)=2.02, \underline{p}>.05$. This means that reversing the film's position had no effect on the infant's performance on the task.

An analysis of variance conducted on the PTLT across the three age groups also revealed no significant differences neither were there significant differences on the first look measure across the groups.

\section{Discussion}

These results indicate that the ability to detect temporal synchrony between pauses in an object's motion and impact sounds does not emerge by 2 1/2-months of age. Taken together the results of this study do indicate a 
developmental trend of infants' sensitivity to temporal synchrony with the ability emerging by 4 months of age and appears to no longer guide visual selectivity at 6 months of age. 


\section{Chapter 4}

\section{General Discussion}

The ability to detect a relationship between an impact sound and synchronous pauses in continuous motion appears to emerge by 4 months of age, however this sensitivity seems to have less influence on visual selectivity by 6 months of age. A possible explanation for these results is that by 6 months of age infants are familiar with synchronous sights and sounds occurring in synchrony and so they found the nonsynchronous presentation more interesting or novel to attend to. Also perhaps by 6 months of age, infants, like adults, are more sensitive to a synchrony relationship involving impacts with a surface. Furthermore, the inability of 2-1/2month-old infants to match sounds and pauses on this task may have been a direct result of the limitations of the 2screen procedure when used with these young infants. In order to perform on a preference task young infants need to already possess attentional mobility and motor coordination, and this is not yet fully developed at $21 / 2$ months of age. Therefore we cannot conclude from this experiment that 2 1/2-month-old infants are unable to detect synchrony because research conducted in our lab has shown that infants at 2 $1 / 2$ months of age and even as young as 3 weeks of age are able to detect synchrony using an infant controlled habituation paradigm. Future research needs to utilize a 
design which capitalizes on young infants' strengths in determining their ability to match a synchronous sight and sound.

Similar findings of infants detecting a given intermodal relationship at an earlier age and not at a later age have been reported by Pickens et al (1992) where they found that fullterm infants displayed evidence of face-voice matching at 3 and 7 months but not at 5 months of age. The authors concluded that the failure of 5-month-old infants to match faces and synchronous voices on this task may have been due to the fact that the face-voice stimuli were not salient enough to produce preferential looking on the task. It is possible that these infants since they demonstrated matching at a younger age are already familiar with synchronized faces and voices and so the non-synchronous face-voice appeared to be more novel and more salient. This may also have been the case with the 6 -month-olds in the current study. Based on these findings, future research should look at infants' performance at 8 months of age on this task to see if at this age looking preferences will once again change from looking to the nonsynchronous to the synchronous event. It seems reasonable to assume that infants' preference would change from nonsynchrony to synchrony because as adults we perceive sounds as unitary events. 
On the other hand, other research has shown that infants at 4 months of age will sometimes look preferentially at a nonsynchronous event rather than a synchronous one. For instance, Bahrick (1983) found that in one experimental condition 4 1/2-month-old infants who were presented with films of the same event side by side along with a synchronized and appropriate soundtrack to one of the events, these infants showed a significant preference for the event that was out of synchrony with the soundtrack they were hearing. However, in other conditions they were able to match the films and soundtracks on the basis of synchrony as well as on the basis of elasticity and/or rigidity of substance.

Bahrick (1988) further found that infants of 3 months of age were unable to relate objects to sounds when the soundtracks were either nonsynchronous or inappropriate to the composition of the object. She only found evidence of intermodal learning when infants were trained with appropriate and synchronous film and soundtrack pairings. This study along with others have shown that there are developmental changes in detection of audiovisual synchrony (Bahrick, 1987; Humphrey \& Tees 1980).

Spelke, Born \& Chu (1983) found that 4-month-old infants perceived correspondences between an object and a sound when there was a pause and change in trajectory of an 
object, regardless of whether the change coincided with an impact against a surface. Future research needs to determine whether infants are able to match a change in trajectory to an impact sound in the absence of a pause. These researchers also found that infants did not match impact sounds with the arrival of an object at a specific spatial location. Future research should address this phenomenon, that is, whether the presence of a marker at the point of impact would enable infants to match on the basis of spatial location. Another idea for future research would be to once again compare an object pausing in midair against one which paused on contact with the surface. However this time films would be used rather than live objects. This procedure would enable better control of the synchrony between the objects and the impact sound. The results of this new experiment might show that infants, like adults, view an object contacting a visible surface as more salient than an object pausing in midair. These results are inconsistent with the integrationassociation view as the theory does not explain how infants are able to match at 4 months of age and not at 6 months of age. This theory posits that through association on the basis of co-occurrence infants learn which sights and sounds belong together. Infants at 4 and 6 months of age had an equal opportunity to learn this relationship however matching was only present in the 4 -month-old group. The 
results are also inconsistent with Piaget's action-centered view as they occur too early in development for motor capabilities to be developed. On the other hand, the pattern of development which was uncovered in this research is consistent with Gibson's (1969) increasing specificity view of perceptual development. Synchrony appears to be important in guiding looking by 4 months of age but by 6 months of age infants are more interested in the differentiation of more specific audible and visible attributes and this may lead them to look at the more novel out of synchrony presentation.

Overall this research adds to our knowledge of infants' detection of audio-visual events and by doing so it provides a preliminary step for determining the basis of adults' detection of audio-visual events. 


\section{References}

Bahrick, L.E. (1983). Infants' perception of substance and temporal synchrony in multimodal events. Infant Behavior and Development, $\underline{6}, 429-451$.

Bahrick, L.E. (1987). Infants' intermodal perception of two levels of temporal structure in natural events. Infant Behavior and Development, 10, 387-416.

Bahrick, L.E. (1988). Intermodal learning in infancy:

Learning on the basis of two kinds of invariant relations in audible and visible events. Child Development, 59, 197-209.

Birch, H.G. \& Lefford, A. (1963). Intersensory development in children. Monographs of the Society for Research in Child Development, 28, \#89.

Bryant, P.(1974). Perception and understanding in young children: An experimental approach: London: Methuen. Dodd, B. (1979). Lip reading in infants: Attention to speech presented in-and-out-of synchrony. Cognitive Psychology, 11, 478-484.

Gibson, E.J. (1969). Principles of perceptual learning and development. New York: Appleton-Century Crofts. Humphrey, K. \& Tees, R. (1980). Auditory-visual coordination in infancy. Some limitations of the preference methodology. Bulletin of the Psychonomic Society, 16, 213-216. 
Humphrey, K., \& Tees, R.C., \& Werker, J. (1979). Auditoryvisual integration in infancy. Developmental Psychology, 16, 185-192.

Lawson, K.R. (1980). Spatial and temporal congruity and auditory visual integration in infancy. Developmental Psychology, 16, 185-192.

Lewkowicz, D.J. (1992, in press). The development of temporally-based intersensory perception in human infants. In F.Macar \& V. Pouthas (Eds.) Time, Action, \& Cognition. Kluwer: Academic Publishers.

Lewkowicz, D.J. (1985). Bisensory response to temporal frequency in 4-month old infants. Developmental

Psychology, $2,306-317$.

Mendelson, M.J. \& Ferland, M.B. (1982). Auditory-visual transfer in four-month-old infants. Child Development, 53, $1022-1027$.

Menten, J.M. \& Cohen, L.B. (Sept. 1979). Infant perception of auditory-visual synchrony. Paper presented at the American Psychological Association Meetings. Pickens, J.N., Field, T., Nawrocki, T., Martinez, A., Soutollo, D., \& Gonzalez, J. (May, 1992). Fullterm and Preterm Infants' Perception of Face-Voice Synchrony. Presented at the International Conference on Infant Studies, Miami Beach, Florida. 
Spelke, E.S. (1976). Infants' intermodal perception of events. Cognitive Psychology, 8, 553-560.

Spelke, E.S. (1979). Perceiving bimodally specified events in infancy. Developmental Psychology, 15, 626-636. Spelke, E.S. (1981). The infant's acquisition of knowledge of bimodally specified events. Journal of Experimental Child Psychology, 31, 279-299.

Spelke, E.S., Born, W.S., \& Chu, F. (1983). Perception of moving sounding objects by four-month-old infants. Perception, 12, 719-732.

Spelke, E.S. \& Cortelyou, A. (1980). Perceptual aspects of social knowing: Looking and listening in infancy. In M.E. Lamb \& Sherrod (Eds.), Infant social cognition (p. 6184). Hillsdale, N.J.: Erlbaum Associates. 
Table 1

PTLT to the Sound Matched Film for Experiment 1

\section{Experiment 1}

$$
\begin{array}{llll}
\mathrm{P} 1 & \mathrm{P} 2 & \mathrm{P} 1+\mathrm{P} 2 / 2 & \mathrm{SEARCH}
\end{array}
$$

$\begin{array}{lrrrr}N & 18 & 18 & 18 & 18 \\ \text { Mean Proportion } & .45 & .45 & .45 & .49 \\ \text { SD } & .27 & .29 & .08 & .17 \\ t & -.77 & -.74 & -2.94 * \star & -.23\end{array}$

4 -month-olds

$\mathrm{N}$

Mean Proportion

SD

$t$

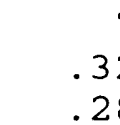

7

$-1.66$

7
.57

.30

.59

$-2.94 * *$

.23

6-month-olds

$\mathrm{N}$

Mean Proportion

SD

$t$

11

11

.37

11

.45

11

.53

.28

.07

$-1.49$

-2.41 *

.45

.09

.48

\section{$-1.49$}

$-1.96$

$* \mathrm{p}<.05$
$* * \mathrm{p}<.01$ 
Table 2

PTLT to the Sound Matched Film for Experiment 2

Experiment 2

$\mathrm{N}=16$

$\begin{array}{lcccc} & & \underline{M} & \underline{\text { SD }} & \underline{t} \\ \text { PTLT } & 1-12 & .49 & .06 & -.94 \\ 1-6 & .49 & .12 & -.42 \\ & 7-12 & .50 & .16 & .13 \\ \text { First Looks } & 1-12 & .46 & .12 & -1.45 \\ & 1-6 & .45 & .16 & -1.31 \\ & 7-12 & .48 & .19 & -0.44 \\ & \text { SEARCH } & .47 & .17 & -0.65\end{array}$




\section{Table 3}

PTLT and First Looks to the sound Matched Film for

Experiment 3

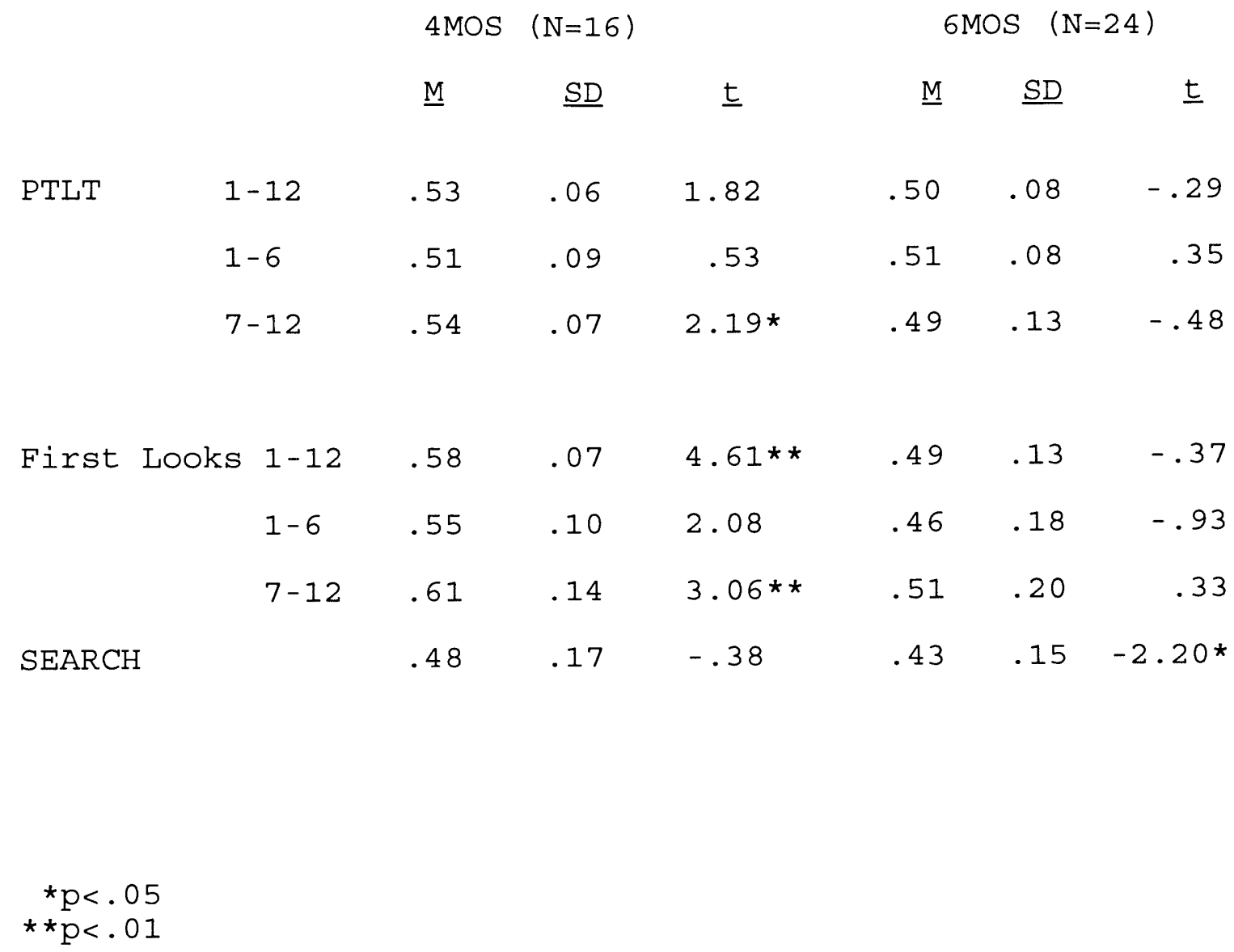




\section{Table 4}

Experiment 3: Mean Proportion of First Looks for 4-montholds who had the Films Reversed versus those who did not

$\begin{array}{lll}1-12 & 1-6 & 7-12\end{array}$

Reversal

$\mathrm{N}$

$\mathrm{M}$

$S D$

$t$

No Reversal

$\mathrm{N}$

Mean Proportion

SD

$t$

$\star \mathrm{p}<.05$

$\star * p<.01$
8
59

.06

4.74 **

.57

.08

2.29

8

8

.58

.09

2.65 *

.52

.11

.55

8

.62

.15

2.26 
Table 5

PTLT and First Looks to the Sound Matched Film for

\section{Experiment 4}

$\mathrm{N}=16$

\begin{tabular}{|c|c|c|c|c|}
\hline \multirow{3}{*}{ PTLT } & & $\underline{\mathrm{M}}$ & $\underline{\mathrm{SD}}$ & $\underline{t}$ \\
\hline & $1-18$ & .48 & .11 & -.80 \\
\hline & $1-6$ & .47 & .20 & -.54 \\
\hline \multirow{6}{*}{$\begin{array}{l}\text { First } \\
\text { Looks }\end{array}$} & $7-12$ & .48 & .11 & -.59 \\
\hline & $13-18$ & .48 & .13 & -.50 \\
\hline & $1-18$ & .52 & .13 & .48 \\
\hline & $1-6$ & .52 & .23 & .33 \\
\hline & $7-12$ & .51 & .17 & .22 \\
\hline & $13-18$ & .50 & .15 & -.04 \\
\hline
\end{tabular}




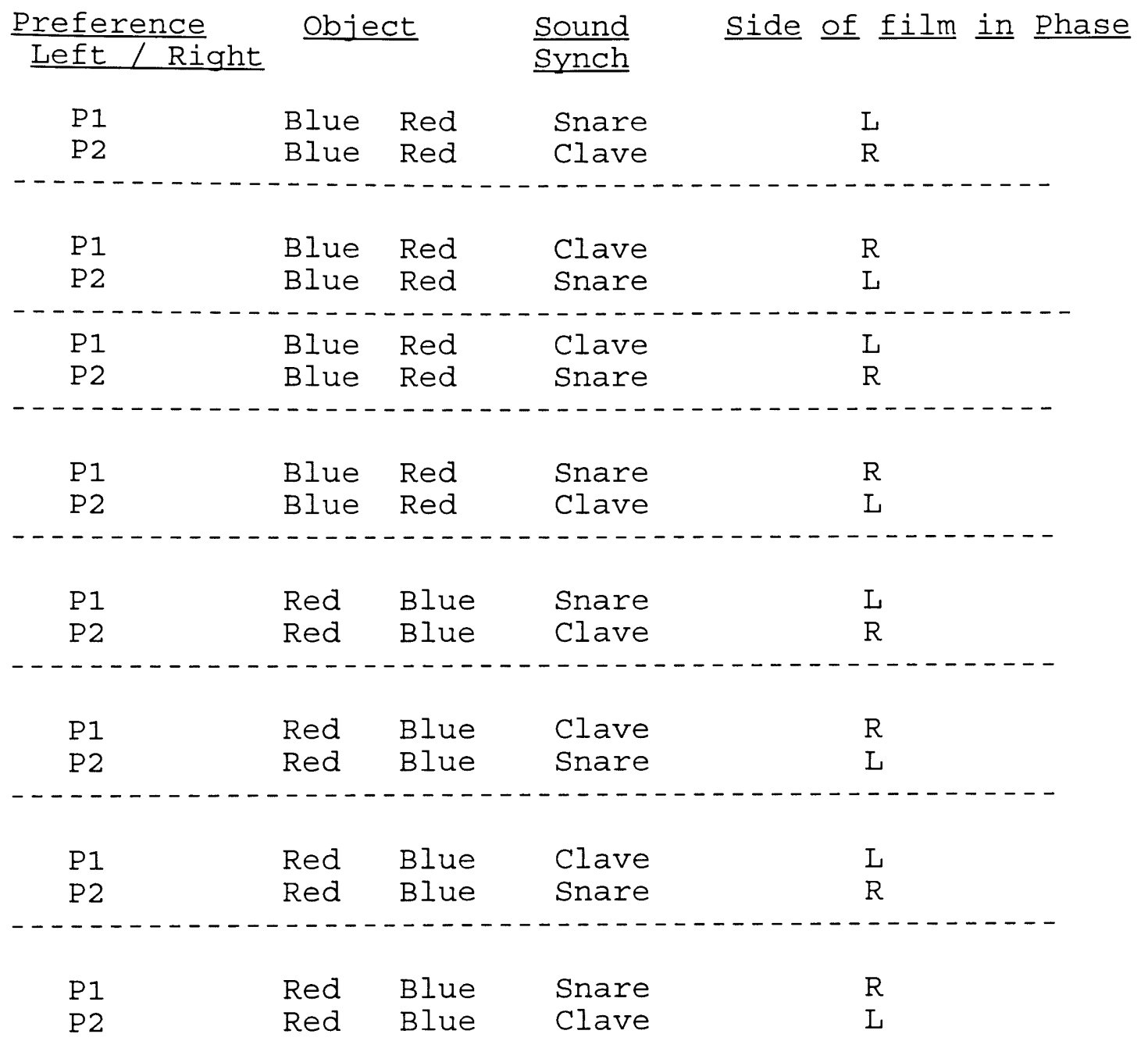

